\title{
Decomposição das taxas de homicídios no Brasil e seus estados: a "demografia" é de fato importante?
}

\author{
Ari Francisco de Araujo Junior ${ }^{*}$ \\ Cláudio Djissey Shikida*
}

\begin{abstract}
RESUMO - Este artigo estuda a relação entre fatores demográficos e taxa de homicídios para o Brasil no período 1996-2007. Como se sabe, a análise do comportamento da taxa de homicídios de forma desagregada nos permite entender melhor a influência de fatores demográficos e fatores específicos da função de mortalidade por homicídios. Para nossa amostra, os resultados mostram que alterações na composição etária não contribuem significativamente na explicação das variações das taxas de homicídios no período.
\end{abstract}

Palavras-chave: Demografia. Homicídios. Criminologia.

\section{INTRODUÇÃO}

Muito tem sido discutido sobre a relação entre a violência e alterações de características demográficas da população em análise. A literatura não é consensual nesse sentido. Alguns autores argumentam que as ondas de crime estão associadas a mudanças importantes na estrutura etária da população (FOX, 1996 e 1997; WILSON, 1994, Scientific American, 1996, apud LEVITT, 1999). Levitt (1999) sugere que mudanças na composição etária apresentam influência limitada sobre as taxas agregadas de crime. Seus resultados indicam que as mudanças na estrutura etária explicam as flutuações das taxas de violência em não mais que 1\% ao ano, o que significaria que as mudanças na estrutura etária esperadas para o caso americano no futuro não trariam contribuição importante para a redução do problema.

De Mello e Schneider (2007) encontram resultados para o estado de São Paulo mais alinhados àqueles resumidos por Fox (Scientific American, 1996, apud LEVITT, 1999). Usando uma base de dados dos municípios paulistas para o período de 1991 a 2005 e regressões que exploram a característica de painel dos dados, em que o logaritmo da taxa de homicídios é o regressando e o logaritmo do percentual da população com idade entre 15 e 24 anos é o regressor (além de outros controles), os autores afirmam que:

Avaliando o efeito da demografia usando a menor estimativa, a mudança demográfica entre a segunda metade dos anos 1990 e a primeira metade dos

\footnotetext{
${ }^{*}$ Mestre em teoria econômica pela Universidade Federal de Minas Gerais. É professor assistente do Ibmec-MG. Endereço eletrônico: arifaj@ibmecmg.br.

** Doutor em economia pela Universidade Federal do Rio Grande do Sul. É professor do Ibmec-MG. Endereço eletrônico: claudiods@ibmecmg.br.
} 
anos 2000 causou uma redução de 7,14\% nos homicídios, ou seja, a demografia responde pela metade da redução dos homicídios entre esses dois períodos. (MELLO; SCHNEIDER, 2007).

A conclusão acima está sujeita a alguns reparos. Primeiro, a taxa de homicídios, como qualquer taxa, é uma média ponderada. Vale lembrar que uma variável construída desta forma está sempre, em tese, sujeita a diversos efeitos de composição (idade, gênero etc.). No caso específico, os autores estão preocupados com a estrutura etária. Ou seja, podemos dizer que a taxa de homicídios é uma média ponderada das taxas específicas de mortes por essa causa em que o peso, em cada ponto, é dado pela própria estrutura etária. Segundo, ainda que se defina (inadequadamente) como efeito da demografia a parcela relativa à estrutura etária, o uso de um grupo etário apenas na regressão não decompõe, no período, a influência da "demografia". Portanto, não há dúvida de que o resultado encontrado representa a contribuição total de um grupo etário específico para a redução da taxa agregada de homicídios. Mas trata-se de um erro conceitual e de interpretação atribuir esse efeito específico ao que de fato observaríamos se pudéssemos estudar o que ocorre para toda a distribuição etária da população' ${ }^{1}$.

$\mathrm{Na}$ verdade, se queremos entender corretamente, do ponto de vista quantitativo, a relação entre as mudanças na estrutura etária e as taxas agregadas de homicídios, deveríamos realizar um exercício de decomposição tal como sugerido por Preston et al. (2001). Esse é o objetivo central do artigo: realizar um exercício de decomposição das taxas agregadas de homicídios de todos os estados do Brasil entre os anos de 1996 a 2007 de modo a quantificar a contribuição das alterações na estrutura etária sobre as flutuações das taxas de homicídios. $\mathrm{Na}$ próxima seção contextualizamos o exercício usando o caso do estado de São Paulo. Na seção seguinte apresentamos a metodologia de decomposição adotada no artigo. Os resultados são discutidos na Seção 4. Na última seção são discutidas as principais conclusões.

\section{CONTEXTUALIZAÇÃO}

No Gráfico 1 apresentamos as variações percentuais das taxas brutas de homicídios para os estados brasileiros entre 1996 e 2007. Os dados sugerem que o problema tem uma ele-

1 Vale ainda um comentário adicional: as regressões de De Mello e Schneider (2007) podem também estar superestimando o efeito da proporção de jovens, pois faltam controles importantes que são omitidos, por exemplo, variáveis que captariam o efeito da repressão/dissuasão nos municípios. Curiosamente, os autores não mostram a irrelevância desses fatores dentro da especificação econométrica que, supostamente, mostraria evidências favoráveis à sua tese. Ao invés disso, há apenas um argumento verbal sobre esses possíveis efeitos. Nesse sentido, os autores trataram ambos os argumentos de forma desigual. Isso não seria um problema sério se os argumentos fossem complementares entre si, mas o procedimento não nos permite julgar a suposta pequena magnitude relativa da repressão/dissuasão sob o mesmo critério. 
vação significativa em alguns estados, por exemplo, mais que $100 \%$ no período, nos seguintes casos: Minas Gerais, Piauí, Maranhão, Pará, Alagoas e Rio Grande do Norte. Em outros, como São Paulo, Amapá, Roraima, Rio de Janeiro, Mato Grosso do Sul, Distrito Federal e Acre, notamos que a variação percentual foi negativa no período.

GRÁFICO 1 - VARIAÇÃO PERCENTUAL DAS TAXAS DE HOMICÍDIOS (1996 A 2007)

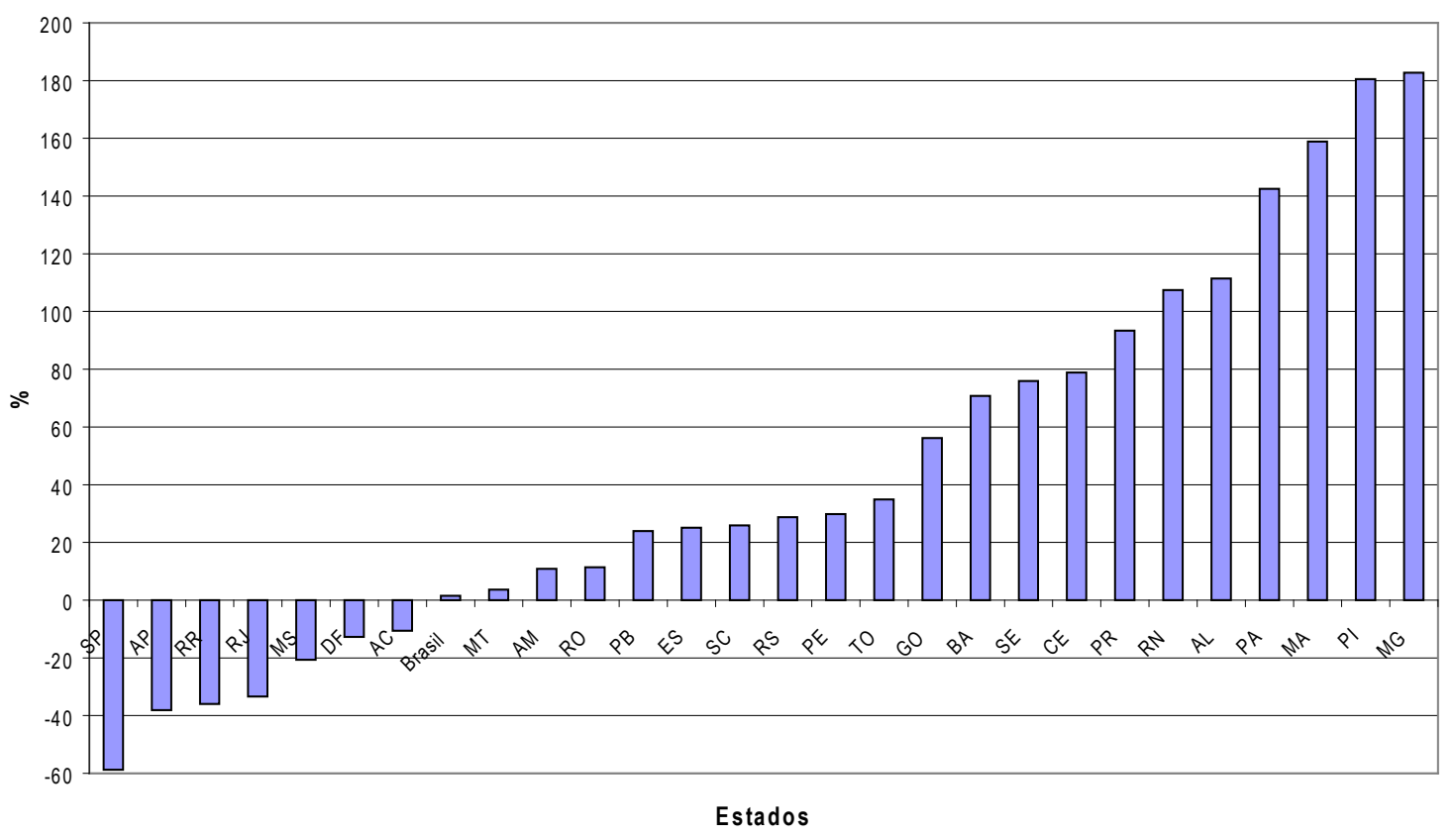

FONTE: Elaboração própria a partir dos dados do DATASUS.

Esses resultados podem ser atribuídos, a partir do foco do artigo, em qualquer dos casos (variações percentuais positivas ou negativas no período), a mudanças na estrutura etária ou da função mortalidade por homicídios. Isso porque, como afirmado anteriormente, uma taxa bruta ou agregada é uma média ponderada tal que:

$$
H R^{t}=\sum_{i}^{\infty} C_{i}^{t} S H R_{i}^{t}
$$

em que $H R^{t}$ é a taxa de homicídios no ano $t, S H R_{i}^{t}$ é a taxa específica (de cada grupo etário) de homicídios (função mortalidade por homicídios) e $C_{i}^{t}$ é o percentual ou peso de cada grupo etário na população (distribuição etária). Fácil notar que a taxa bruta de homicídios pode variar ou por mudanças na função mortalidade ou por mudanças na estrutura etária (ou em ambas).

Vamos utilizar aqui o exemplo do estado de São Paulo. Vimos que São Paulo apresentou, no período entre 1996 e 2007, uma redução de 58,8\% nas taxas de homicídios (a taxa bruta de mortalidade de 1996 é de 36,30 por 100 mil enquanto a de 2007 é de 14,96 por 100 mil). A 
pergunta relevante é: o que explica essa queda? A resposta: mudança nas taxas específicas, nos pesos ou ambas. Podemos notar, pelo Gráfico 2, que a função mortalidade do estado de São Paulo sofre, com exceção do grupo etário 75-79 anos, um deslocamento para baixo.

GRÁFICO 2 - TAXAS ESPECÍFICAS DE MORTALIDADE POR HOMICÍDIOS (SHRI) - SÃO PAULO (1996 A 2007)

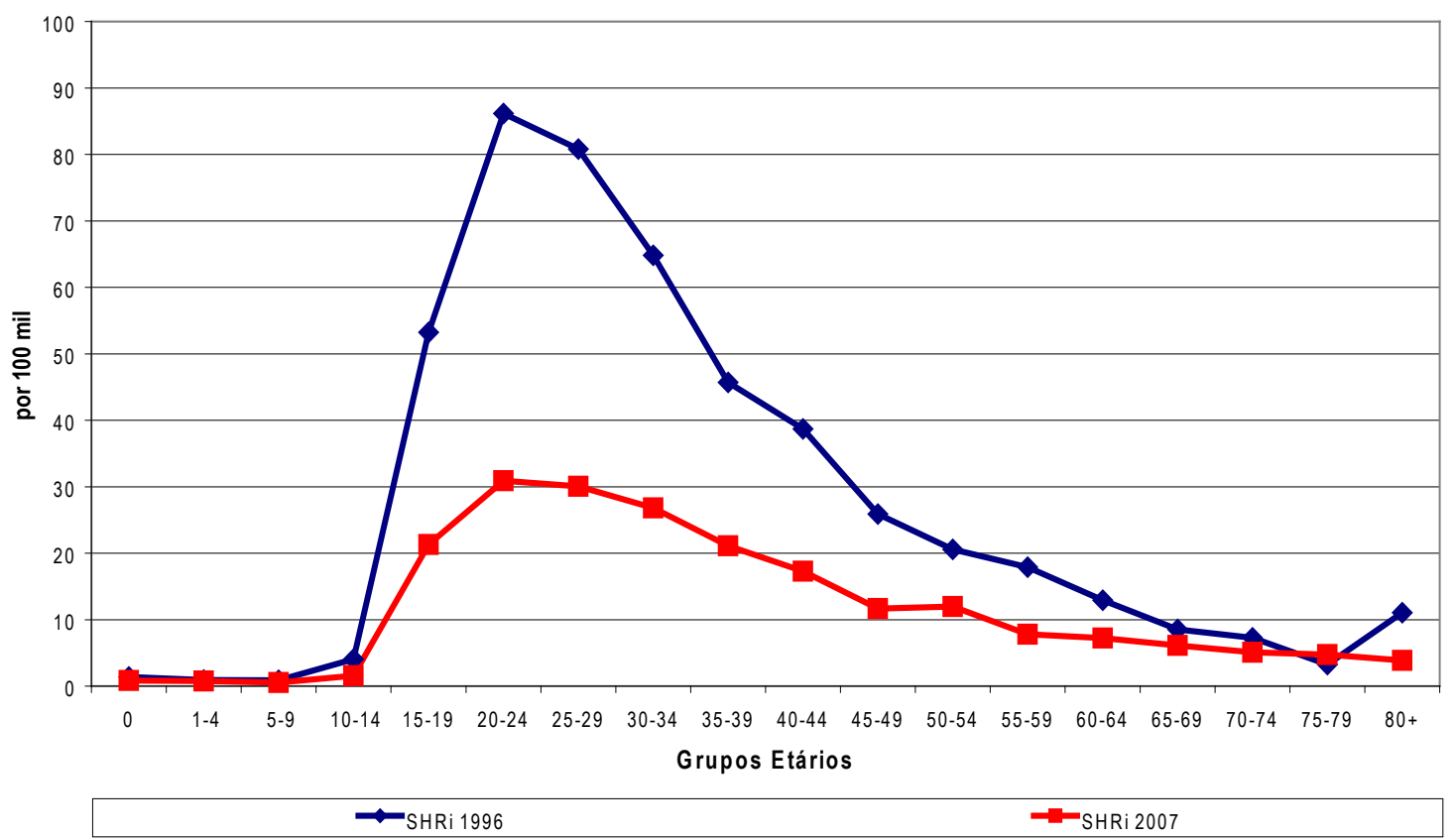

FONTE: Elaboração própria a partir dos dados do DATASUS.

GRÁFICO 3 - ESTRUTURA ETÁRIA DA POPULAÇÃO (CI) - SÃO PAULO (1996 A 2007)

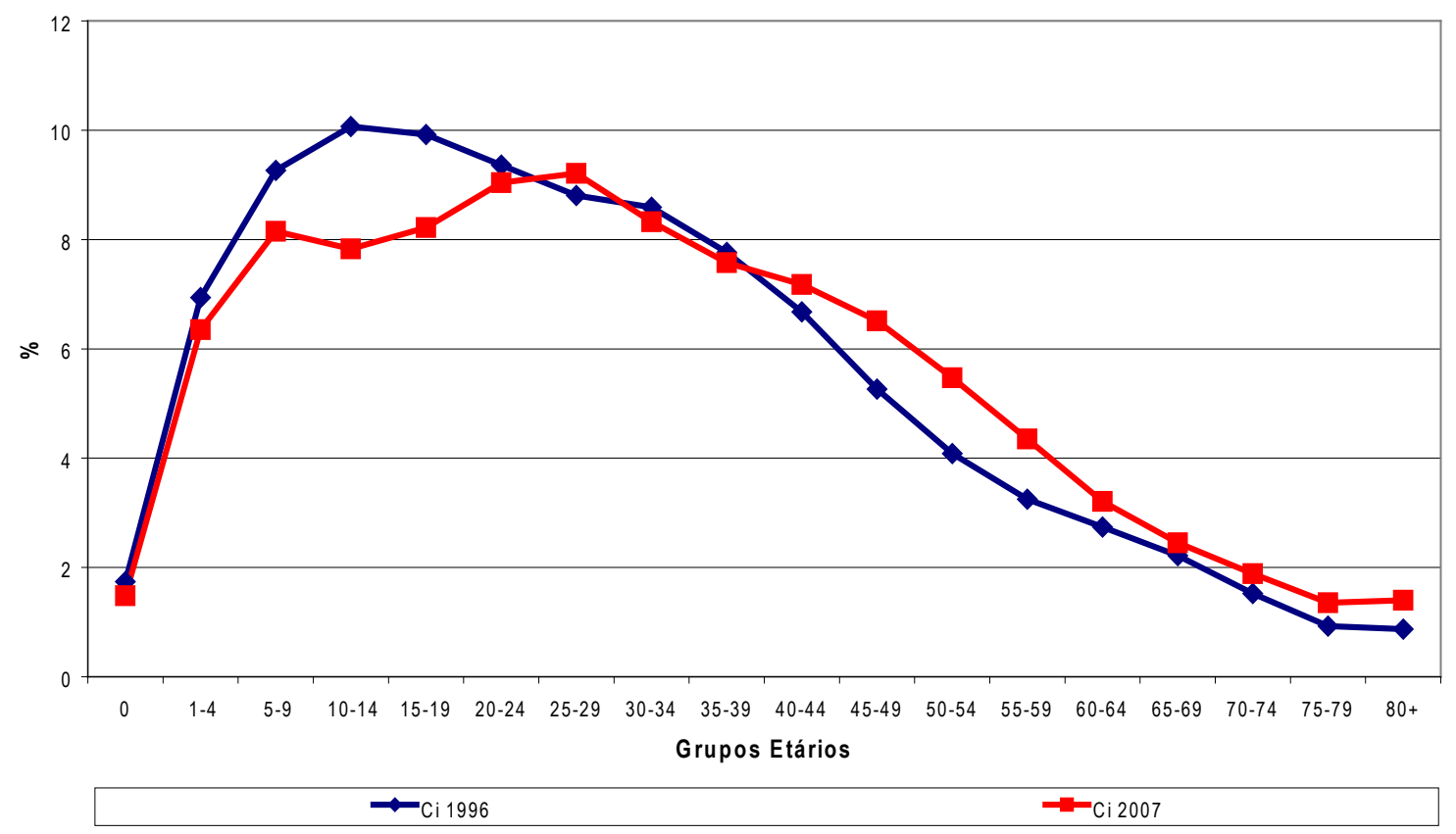

FONTE: Elaboração própria a partir dos dados do DATASUS.

90 
Por outro lado, pelo Gráfico 3, em que a estrutura etária de 1996 e 2007 da população paulista é apresentada, podemos notar que ocorre uma alteração nos pesos de cada grupo etário. Tal como esperado, temos uma redução do peso dos grupos mais jovens e um aumento daqueles de idades mais avançadas. Trata-se de uma estrutura em envelhecimento típica de região em estágio mais avançado da transição demográfica. A dúvida que fica é: qual contribuição é maior, aquela observada pelas mudanças na função mortalidade ou a observada nas alterações da distribuição etária da população? Claro que, se a contribuição da função mortalidade for relativamente maior do que a estimada para as alterações na estrutura etária, as variáveis que afetam a função mortalidade do crime, condições econômicas, sociais e de dissuasão, são as maiores suspeitas para o estudo dos principais determinantes da taxa de homicídios. Ou seja, a "demografia" contribuiria menos para a queda do que os outros determinantes também consagrados na visão da economia da criminalidade (BECKER, 1968).

O fato é que, quando comparamos uma taxa bruta de um período contra outra de um período adiante, temos que ter claro que a taxa bruta mais recente pode ser maior ou menor que aquela observada anteriormente devido a uma combinação de mudanças nas taxas específicas e na distribuição etária da população. Na próxima seção apresentamos uma metodologia para a realização de um exercício de decomposição das contribuições de cada dimensão (mortalidade e estrutura etária).

\section{METODOLOGIA E DADOS}

\subsection{DECOMPOSIÇÃO DAS DIFERENÇAS ENTRE TAXAS}

A metodologia usada aqui é uma adaptação daquela apresentada em Preston et al. (2001). Preston (2001) apresenta a metodologia, devida a Kitagawa (1955), de decomposição entre taxas de unidades territoriais em um mesmo período. O que fazemos aqui é apenas uma variante, ou seja, a decomposição das taxas de uma mesma unidade territorial (no caso, cada estado brasileiro) em dois pontos no tempo. A variação da taxa de homicídios de um estado qualquer no tempo pode ser escrita da seguinte forma:

$$
\Delta=H R^{t}-H R^{t-1}=\sum_{i} C_{i}^{t} S H R_{i}^{t}-\sum_{i} C_{i}^{t-1} S H R_{i}^{t-1}
$$

A Equação (1) pode ser reescrita tal que (dividindo cada um dos termos em duas partes iguais e somando dois termos iguais a zero): 


$$
\begin{aligned}
\Delta & =\frac{\sum_{i} C_{i}^{t} S H R_{i}^{t}}{2}+\frac{\sum_{i} C_{i}^{t} S H R_{i}^{t}}{2}-\frac{\sum_{i} C_{i}^{t-1} S H R_{i}^{t-1}}{2}-\frac{\sum_{i} C_{i}^{t-1} S H R_{i}^{t-1}}{2}+ \\
& +\frac{\sum_{i} C_{i}^{t} S H R_{i}^{t-1}}{2}-\frac{\sum_{i} C_{i}^{t} S H R_{i}^{t-1}}{2}+\frac{\sum_{i} C_{i}^{t-1} S H R_{i}^{t}}{2}-\frac{\sum_{i} C_{i}^{t-1} S H R_{i}^{t}}{2}
\end{aligned}
$$

Uma manipulação algébrica adicional deixa (3) como (combinando os oito termos em quatro):

$$
\begin{aligned}
\Delta= & \sum_{i} C_{i}^{t}\left[\frac{S H R_{i}^{t}+S H R_{i}^{t-1}}{2}\right]-\sum_{i} C_{i}^{t-1}\left[\frac{S H R_{i}^{t}+S H R_{i}^{t-1}}{2}\right]+ \\
& +\sum_{i} \operatorname{SHR}_{i}^{t}\left[\frac{C_{i}^{t}+C_{i}^{t-1}}{2}\right]-\sum_{i} \operatorname{SHR}_{i}^{t-1}\left[\frac{C_{i}^{t}+C_{i}^{t-1}}{2}\right]
\end{aligned}
$$

Finalmente temos (e em dois termos):

$$
\Delta=\sum_{i}\left(C_{i}^{t}-C_{i}^{t-1}\right)\left[\frac{S H R_{i}^{t}+S H R_{i}^{t-1}}{2}\right]+\sum_{i}\left(S H R_{i}^{t}-S H R_{i}^{t-1}\right)\left[\frac{C_{i}^{t}+C_{i}^{t-1}}{2}\right]
$$

em que:

$$
\begin{aligned}
& \sum_{i}\left(C_{i}^{t}-C_{i}^{t-1}\right)\left[\frac{S H R_{i}^{t}+S H R_{i}^{t-1}}{2}\right] \text { é a contribuição da composição etária e, } \\
& \sum_{i}\left(S H R_{i}^{t}-S H R_{i}^{t-1}\right)\left[\frac{C_{i}^{t}+C_{i}^{t-1}}{2}\right] \text { é a contribuição do esquema de taxas específicas. }
\end{aligned}
$$

Pela Equação (5) podemos, desta forma, separar a importância relativa das mudanças na função mortalidade e na estrutura etária. Os resultados serão apresentados após uma breve apresentação dos dados utilizados. Vale ressaltar que nosso estudo se limita à decomposição. Vários trabalhos elegeram os determinantes da função mortalidade como objeto de estudo (ver, para o caso brasileiro, por exemplo, a revisão de Dos Santos e Kassouf, 2008). 


\subsection{DADOS}

Os dados de mortalidade geral e população residente em 1991 e 2000 foram retirados do site do DATASUS (fonte primária - Declarações de Óbitos e Censos Demográficos) para todos os estados do Brasil.

A mortalidade por homicídios dos estados foi ajustada proporcionalmente devido à existência de casos ignorados por idade. As taxas brutas/agregadas foram calculadas como razões entre o número de ocorrências e o número de grupos de 100 mil habitantes. O mesmo vale para as taxas específicas, nesse caso o cálculo foi feito para cada grupo etário.

\section{RESULTADOS}

A Tabela 1 apresenta os resultados da decomposição da diferença das taxas brutas do Brasil como um todo. Podemos notar que a diferença total positiva de 0,37 mortes para cada 100 mil representa, a partir da metodologia utilizada, 0,86 devido a mudanças na estrutura etária e -0,49 devido a alterações nas condições de mortalidade, nesse caso, em sentido contrário. Isso significa que, para o caso do Brasil, apesar da contribuição favorável da função mortalidade, a estrutura etária exerce impacto suficiente para compensar esse efeito e ainda produzir um efeito líquido que reflete numa elevação da taxa bruta de homicídios no Brasil entre 1996 e 2007.

TABELA 1 - DECOMPOSIÇÃO DAS DIFERENÇAS ENTRE AS TAXAS: BRASIL (1996) X BRASIL (2007)

\begin{tabular}{|c|c|c|c|c|}
\hline \multirow{2}{*}{$\begin{array}{l}\text { Grupo } \\
\text { Etário }\end{array}$} & \multicolumn{2}{|c|}{ a } & \multicolumn{2}{|c|}{ b } \\
\hline & \multicolumn{2}{|c|}{ BR 1996} & \multicolumn{2}{|c|}{ BR 2007} \\
\hline i & Ci & Mi & $\mathbf{C i}$ & $\mathrm{Mi}$ \\
\hline 0 & 0.019835 & 2.850875 & 0.016832 & 2.470500 \\
\hline $1-4$ & 0.079897 & 0.907580 & 0.070536 & 0.727341 \\
\hline $5-9$ & 0.104660 & 0.794538 & 0.089637 & 0.765137 \\
\hline $10-14$ & 0.111809 & 3.052302 & 0.088255 & 3.542934 \\
\hline $15-19$ & 0.106464 & 33.355086 & 0.091197 & 43.156891 \\
\hline $20-24$ & 0.091971 & 56.766806 & 0.095307 & 57.722745 \\
\hline $25-29$ & 0.082775 & 52.706088 & 0.090793 & 51.313731 \\
\hline $30-34$ & 0.079097 & 44.761191 & 0.078386 & 40.964873 \\
\hline $35-39$ & 0.069475 & 36.597204 & 0.070427 & 32.259400 \\
\hline $40-44$ & 0.058974 & 30.626612 & 0.066428 & 25.462272 \\
\hline $45-49$ & 0.047526 & 24.509952 & 0.058711 & 20.318849 \\
\hline $50-54$ & 0.037460 & 19.605964 & 0.048487 & 17.118671 \\
\hline $55-59$ & 0.030914 & 16.612933 & 0.038852 & 13.566280 \\
\hline $60-64$ & 0.025487 & 12.972660 & 0.029543 & 11.132521 \\
\hline $65-69$ & 0.020622 & 10.451981 & 0.023109 & 9.978731 \\
\hline $70-74$ & 0.014470 & 8.735206 & 0.017629 & 8.424338 \\
\hline $75-79$ & 0.009286 & 7.521988 & 0.012395 & 7.886253 \\
\hline \multirow[t]{3}{*}{$80+$} & 0.009279 & 10.180102 & 0.013477 & 7.172941 \\
\hline & $\mathrm{TB}$ & 24.83 & TB & 25.20 \\
\hline & & & & 0.37 \\
\hline
\end{tabular}

FONTE: Elaboração própria a partir dos dados do DATASUS. 
TABELA 1 - DECOMPOSIÇÃO DAS DIFERENÇAS ENTRE AS TAXAS: BRASIL (1996) X BRASIL (2007) - CONTINUAÇÃO

\begin{tabular}{|c|c|c|c|c|c|c|}
\hline $\begin{array}{l}\text { Grupo } \\
\text { Etário }\end{array}$ & $\mathrm{Cb}-\mathrm{Ca}$ & $\frac{(\mathrm{Mb}+\mathrm{Ma})}{2}$ & $\begin{array}{l}\text { Contribuição da } \\
\text { composição por } \\
\text { idade: } \\
\text { Cb-Ca* } \frac{(\mathrm{Mb}+\mathrm{Ma})}{2}\end{array}$ & Mb-Ma & $\frac{(\mathrm{Ca}-\mathrm{Cb})}{2}$ & $\begin{array}{l}\text { Contribuição do } \\
\text { esquema de taxas } \\
\text { de mortalidade: } \\
\text { Mb-Ma* } \frac{(\mathrm{Ca}-\mathrm{Cb})}{2}\end{array}$ \\
\hline 0 & -0.003003 & 2.660687 & -0.007990 & -0.380375 & 0.018333 & -0.006974 \\
\hline $1-4$ & -0.009361 & 0.817461 & -0.007652 & -0.180238 & 0.075216 & -0.013557 \\
\hline $5-9$ & -0.015023 & 0.779838 & -0.011715 & -0.029401 & 0.097149 & -0.002856 \\
\hline $10-14$ & -0.023554 & 3.297618 & -0.077671 & 0.490632 & 0.100032 & 0.049079 \\
\hline $15-19$ & -0.015267 & 38.255989 & -0.584036 & 9.801806 & 0.098831 & 0.968720 \\
\hline $20-24$ & 0.003336 & 57.244775 & 0.190959 & 0.955938 & 0.093639 & 0.089513 \\
\hline $25-29$ & 0.008018 & 52.009910 & 0.417021 & -1.392357 & 0.086784 & -0.120834 \\
\hline $30-34$ & -0.000711 & 42.863032 & -0.030477 & -3.796318 & 0.078741 & -0.298927 \\
\hline $35-39$ & 0.000952 & 34.428302 & 0.032782 & -4.337804 & 0.069951 & -0.303435 \\
\hline $40-44$ & 0.007454 & 28.044442 & 0.209048 & -5.164340 & 0.062701 & -0.323807 \\
\hline $45-49$ & 0.011185 & 22.414401 & 0.250714 & -4.191103 & 0.053119 & -0.222625 \\
\hline $50-54$ & 0.011027 & 18.362317 & 0.202489 & -2.487293 & 0.042974 & -0.106888 \\
\hline $55-59$ & 0.007938 & 15.089607 & 0.119779 & -3.046653 & 0.034883 & -0.106277 \\
\hline $60-64$ & 0.004056 & 12.052591 & 0.048884 & -1.840140 & 0.027515 & -0.050631 \\
\hline $65-69$ & 0.002487 & 10.215356 & 0.025402 & -0.473250 & 0.021865 & -0.010348 \\
\hline $70-74$ & 0.003159 & 8.579772 & 0.027102 & -0.310868 & 0.016049 & -0.004989 \\
\hline $75-79$ & 0.003108 & 7.704121 & 0.023947 & 0.364265 & 0.010840 & 0.003949 \\
\hline $80+$ & 0.004197 & 8.676522 & 0.036417 & -3.007161 & 0.011378 & -0.034216 \\
\hline
\end{tabular}

FONTE: Elaboração própria a partir dos dados do DATASUS.

0.369898

TABELA 2 - DECOMPOSIÇÃO DAS DIFERENÇAS ENTRE AS TAXAS: SÃO PAULO (1996) X SÃO PAULO (2007)

\begin{tabular}{|c|c|c|c|c|}
\hline \multirow{2}{*}{$\begin{array}{l}\text { Grupo } \\
\text { Etário }\end{array}$} & \multicolumn{2}{|c|}{$\mathbf{a}$} & \multicolumn{2}{|c|}{ b } \\
\hline & \multicolumn{2}{|c|}{ SP 1996} & \multicolumn{2}{|c|}{ SP 2007} \\
\hline i & $\mathbf{C i}$ & Mi & $\mathbf{C i}$ & Mi \\
\hline 0 & 0.017372 & 1.381415 & 0.014843 & 0.829135 \\
\hline $1-4$ & 0.069394 & 0.907786 & 0.063501 & 0.775224 \\
\hline $5-9$ & 0.092646 & 0.874222 & 0.081516 & 0.543508 \\
\hline $10-14$ & 0.100669 & 4.052546 & 0.078303 & 1.571710 \\
\hline $15-19$ & 0.099211 & 53.245358 & 0.082194 & 21.291572 \\
\hline $20-24$ & 0.093633 & 86.147978 & 0.090418 & 30.897182 \\
\hline $25-29$ & 0.088054 & 80.807234 & 0.092091 & 30.041774 \\
\hline $30-34$ & 0.085914 & 64.803637 & 0.083238 & 26.820399 \\
\hline $35-39$ & 0.077638 & 45.708387 & 0.075782 & 21.111801 \\
\hline $40-44$ & 0.066748 & 38.694717 & 0.071790 & 17.280091 \\
\hline $45-49$ & 0.052622 & 25.880597 & 0.065105 & 11.682102 \\
\hline $50-54$ & 0.040845 & 20.563917 & 0.054720 & 11.965057 \\
\hline $55-59$ & 0.032496 & 17.908235 & 0.043524 & 7.804194 \\
\hline $60-64$ & 0.027410 & 12.914066 & 0.032084 & 7.211333 \\
\hline $65-69$ & 0.022152 & 8.531232 & 0.024487 & 6.131658 \\
\hline $70-74$ & 0.015242 & 7.281992 & 0.018853 & 5.091618 \\
\hline $75-79$ & 0.009256 & 3.240729 & 0.013539 & 4.726910 \\
\hline $80+$ & 0.008701 & 11.032836 & 0.014012 & 3.864519 \\
\hline & TB & 36.30 & $\mathrm{~TB}$ & 14.96 \\
\hline
\end{tabular}

FONTE: Elaboração própria a partir dos dados do DATASUS. 
TABELA 2 - DECOMPOSIÇÃO DAS DIFERENÇAS ENTRE AS TAXAS: SÃO PAULO (1996) X SÃO PAULO (2007) - CONTINUAÇÃO

\begin{tabular}{|c|c|c|c|c|c|c|}
\hline $\begin{array}{l}\text { Grupo } \\
\text { Etário }\end{array}$ & $\mathrm{Cb}-\mathrm{Ca}$ & $\frac{(\mathrm{Mb}+\mathrm{Ma})}{2}$ & \begin{tabular}{|c|}
$\begin{array}{c}\text { Contribuição da } \\
\text { composição por } \\
\text { idade: }\end{array}$ \\
$\begin{array}{c}\mathrm{Cb}-\mathrm{Ca} * \frac{(\mathrm{Mb}+\mathrm{Ma})}{2} \\
\end{array}$
\end{tabular} & Mb-Ma & $\frac{(\mathrm{Ca}-\mathrm{Cb})}{2}$ & \begin{tabular}{|c} 
Contribuição do \\
esquema de taxas \\
de mortalidade: \\
Mb-Ma*(Ca-Cb) \\
2
\end{tabular} \\
\hline 0 & -0.002529 & 1.105275 & -0.002795 & -0.552280 & 0.016108 & -0.008896 \\
\hline $1-4$ & -0.005893 & 0.841505 & -0.004959 & -0.132561 & 0.066447 & -0.008808 \\
\hline $5-9$ & -0.011129 & 0.708865 & -0.007889 & -0.330714 & 0.087081 & -0.028799 \\
\hline $10-14$ & -0.022366 & 2.812128 & -0.062897 & -2.480836 & 0.089486 & -0.221999 \\
\hline $15-19$ & -0.017017 & 37.268465 & -0.634209 & -31.953786 & 0.090703 & -2.898293 \\
\hline $20-24$ & -0.003215 & 58.522580 & -0.188151 & -55.250796 & 0.092026 & -5.084486 \\
\hline $25-29$ & 0.004038 & 55.424504 & 0.223781 & -50.765460 & 0.090073 & -4.572576 \\
\hline $30-34$ & -0.002676 & 45.812018 & -0.122589 & -37.983238 & 0.084576 & -3.212459 \\
\hline $35-39$ & -0.001856 & 33.410094 & -0.061997 & -24.596586 & 0.076710 & -1.886800 \\
\hline $40-44$ & 0.005042 & 27.987404 & 0.141120 & -21.414626 & 0.069269 & -1.483362 \\
\hline $45-49$ & 0.012483 & 18.781350 & 0.234456 & -14.198496 & 0.058863 & -0.835773 \\
\hline $50-54$ & 0.013875 & 16.264487 & 0.225672 & -8.598860 & 0.047782 & -0.410873 \\
\hline $55-59$ & 0.011028 & 12.856214 & 0.141776 & -10.104042 & 0.038010 & -0.384056 \\
\hline $60-64$ & 0.004675 & 10.062700 & 0.047039 & -5.702733 & 0.029747 & -0.169638 \\
\hline $65-69$ & 0.002335 & 7.331445 & 0.017117 & -2.399575 & 0.023319 & -0.055956 \\
\hline $70-74$ & 0.003612 & 6.186805 & 0.022344 & -2.190374 & 0.017048 & -0.037340 \\
\hline $75-79$ & 0.004282 & 3.983819 & 0.017060 & 1.486181 & 0.011397 & 0.016939 \\
\hline $80+$ & 0.005312 & 7.448677 & 0.039565 & -7.168316 & 0.011356 & -0.081406 \\
\hline
\end{tabular}

FONTE: Elaboração própria a partir dos dados do DATASUS.

$-21.340139$

Em especial, o caso de São Paulo é interessante e os resultados estão reportados na Tabela 2. A redução da taxa é toda explicada pelas alterações na função mortalidade, ou seja, a contribuição das alterações na estrutura etária é nula. Em que medida nosso resultado pode confrontar aquele encontrado por De Mello e Schneider (2007)? Vale lembrar que os autores definem (de forma equivocada) o efeito demográfico como o efeito combinado de esquemas de mortalidade/estrutura etária de um grupo etário específico (15-24 anos). Pela definição dos autores, $50 \%$ da redução das taxas de homicídios em São Paulo pode ser explicada pelo que chamam de "demografia".

Como dissemos anteriormente, essa definição não representa adequadamente o efeito da demografia (ou mais precisamente das alterações na estrutura etária da população). O efeito encontrado é a contribuição total (idade/mortalidade) e específica de determinado grupo etário. Vale notar que, se somarmos nossos resultados relativos aos grupos etários de 15-19 e 20-24 anos encontramos exatamente $-8,80$ [-3,53 + (-5,27)], o que representa $-41,3 \%$, algo próximo do valor de De Mello e Schneider (2007). Ou seja, os autores superestimam o efeito da "demografia". Vale reforçar, nesse caso, que a contribuição das alterações na composição etária é nula. Ocorre, liquidamente, uma compensação dos efeitos específicos entre os diversos 
grupos etários. Nesse sentido, nossos resultados estão mais alinhados àqueles apresentados em Levitt (1999).

TABELA 3 - RESULTADOS PARA BRASIL E ESTADOS (PERÍODO 1996-2007)

\begin{tabular}{|c|c|c|c|c|}
\hline & $\begin{array}{l}\text { Taxa de Homicídios } \\
\text { no inicío do período }\end{array}$ & $\begin{array}{c}\text { Variação da Taxa } \\
\text { de Homicídios }\end{array}$ & $\begin{array}{c}\text { Contribuição de alterações } \\
\text { na Estrutura Etária para a } \\
\text { variação no período }\end{array}$ & $\begin{array}{c}\text { Contribuição de alterações na } \\
\text { Função Mortalidade para a } \\
\text { variação no período }\end{array}$ \\
\hline$\overline{\mathrm{BR}}$ & 24.83 & 0.37 & 0.87 & -0.50 \\
\hline SP & 36.30 & -21.34 & 0.02 & -21.36 \\
\hline RJ & 60.24 & -20.12 & -1.56 & -18.56 \\
\hline MG & 7.36 & 13.45 & 0.35 & 13.09 \\
\hline $\mathrm{ES}$ & 42.82 & 10.74 & 1.56 & 9.18 \\
\hline PR & 15.31 & 14.29 & 0.41 & 13.89 \\
\hline SC & 8.30 & 2.15 & 0.39 & 1.75 \\
\hline RS & 15.24 & 4.38 & 0.30 & 4.08 \\
\hline $\mathrm{BA}$ & 15.03 & 10.63 & 1.61 & 9.02 \\
\hline MA & 6.73 & 10.70 & 1.39 & 9.31 \\
\hline AL & 28.19 & 31.42 & 2.28 & 29.14 \\
\hline SE & 14.71 & 11.16 & 1.29 & 9.87 \\
\hline $\mathrm{RN}$ & 9.29 & 9.97 & 1.11 & 8.87 \\
\hline PB & 19.03 & 4.55 & 1.79 & 2.76 \\
\hline PE & 40.89 & 12.19 & 2.76 & 9.44 \\
\hline PI & 4.72 & 8.52 & 0.98 & 7.54 \\
\hline $\mathrm{CE}$ & 12.99 & 10.24 & 1.53 & 8.71 \\
\hline $\mathrm{RO}$ & 24.56 & 2.80 & 2.27 & 0.52 \\
\hline $\mathrm{AC}$ & 21.15 & -2.25 & 1.27 & -3.51 \\
\hline $\mathrm{AM}$ & 18.92 & 2.06 & 1.28 & 0.78 \\
\hline RR & 43.60 & -15.67 & 1.87 & -17.54 \\
\hline $\mathrm{AP}$ & 43.40 & -16.54 & 1.00 & -17.54 \\
\hline PA & 12.54 & 17.87 & 1.84 & 16.02 \\
\hline TO & 12.22 & 4.26 & 1.42 & 2.84 \\
\hline MT & 29.57 & 1.08 & 2.25 & -1.17 \\
\hline MS & 37.81 & -7.82 & 1.64 & -9.47 \\
\hline $\mathrm{GO}$ & 15.64 & 8.78 & 0.64 & 8.13 \\
\hline DF & 38.36 & -4.88 & -0.67 & -4.21 \\
\hline
\end{tabular}

FONTE: Elaboração própria a partir dos dados do DATASUS.

Os resultados para os outros estados estão resumidos na Tabela 3. Podemos notar que a contribuição das alterações na estrutura etária é, de modo geral, pequena. O estado que mais se beneficiou das mudanças na composição etária em termos absolutos foi o Rio de Janeiro. Mas vale notar que $92,2 \%$ da queda é devida ao esquema de taxas de mortalidade por homicídios (explicadas pelas condições econômicas, sociais e dissuasão que, com certeza, evoluíram no período de uma forma combinada/líquida favorável). Relativamente, o Distrito Federal tem a maior queda explicada pelas mudanças na estrutura etária, 13,8\% (dos -4,88 totais em termos absolutos).

Em todos os outros estados a contribuição líquida da mudança da composição etária é positiva, ou seja, caso a contribuição das taxas específicas fosse nula, observaríamos uma elevação da taxa bruta de homicídios. Claro que, nos casos em que isso não ocorre, temos uma 
contribuição favorável da mudança do esquema de taxas de mortalidade que compensa o efeito da distribuição etária (por exemplo, São Paulo, Acre, Roraima, Amapá e Mato Grosso do Sul).

GRÁFICO 4 - VARIAÇÃO NAS TAXAS DE HOMICÍDIOS X CONTRIBUIÇÃO DA ESTRUTURA ETÁRIA - ESTADOS BRASILEIROS (1996 A 2007)

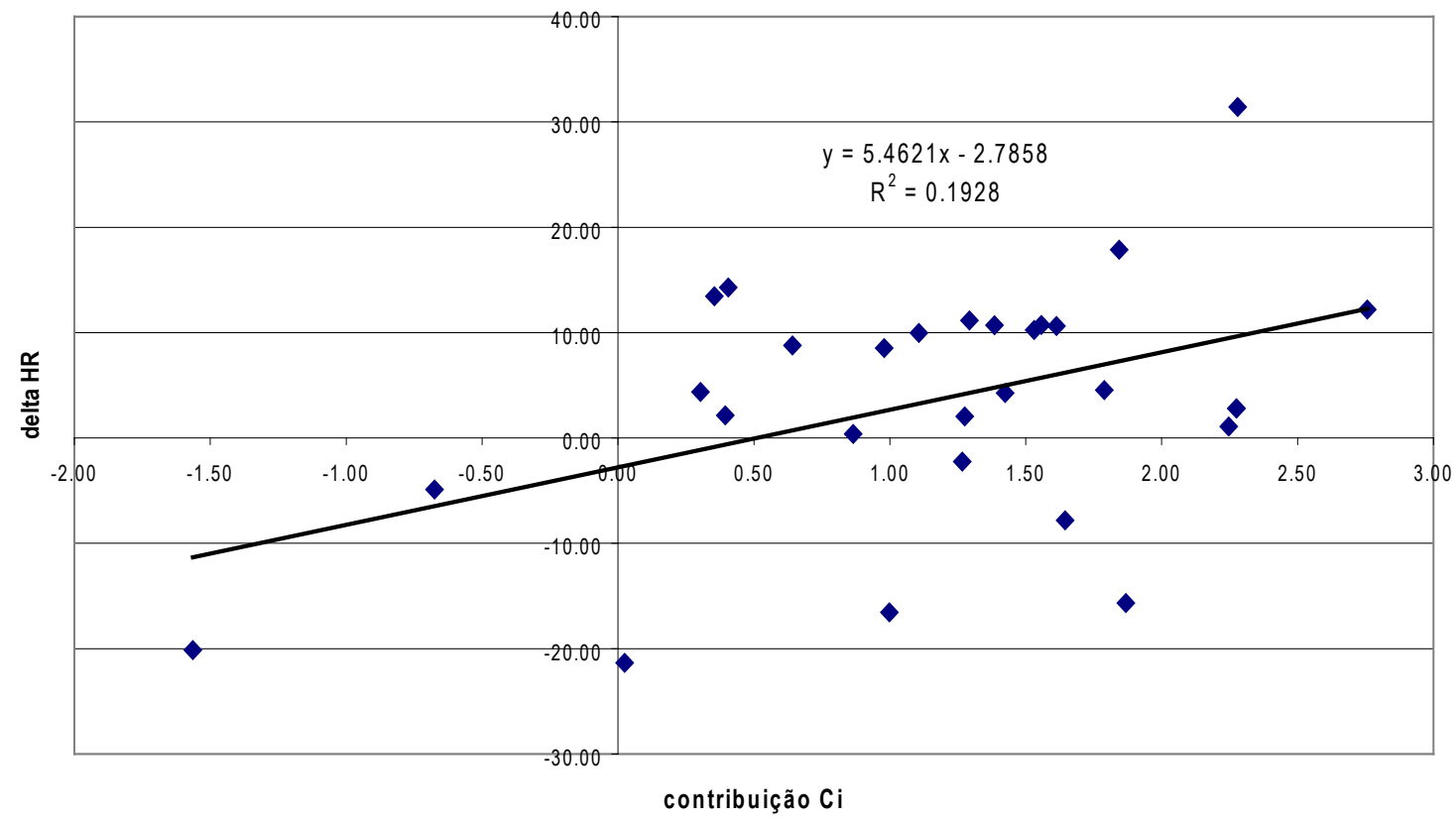

FONTE: Elaboração própria a partir dos dados do DATASUS.

GRÁFICO 5 - VARIAÇÃO NAS TAXAS DE HOMICÍDIOS X CONTRIBUIÇÃO DA FUNÇÃO MORTALIDADE - ESTADOS BRASILEIROS (1996 A 2007)

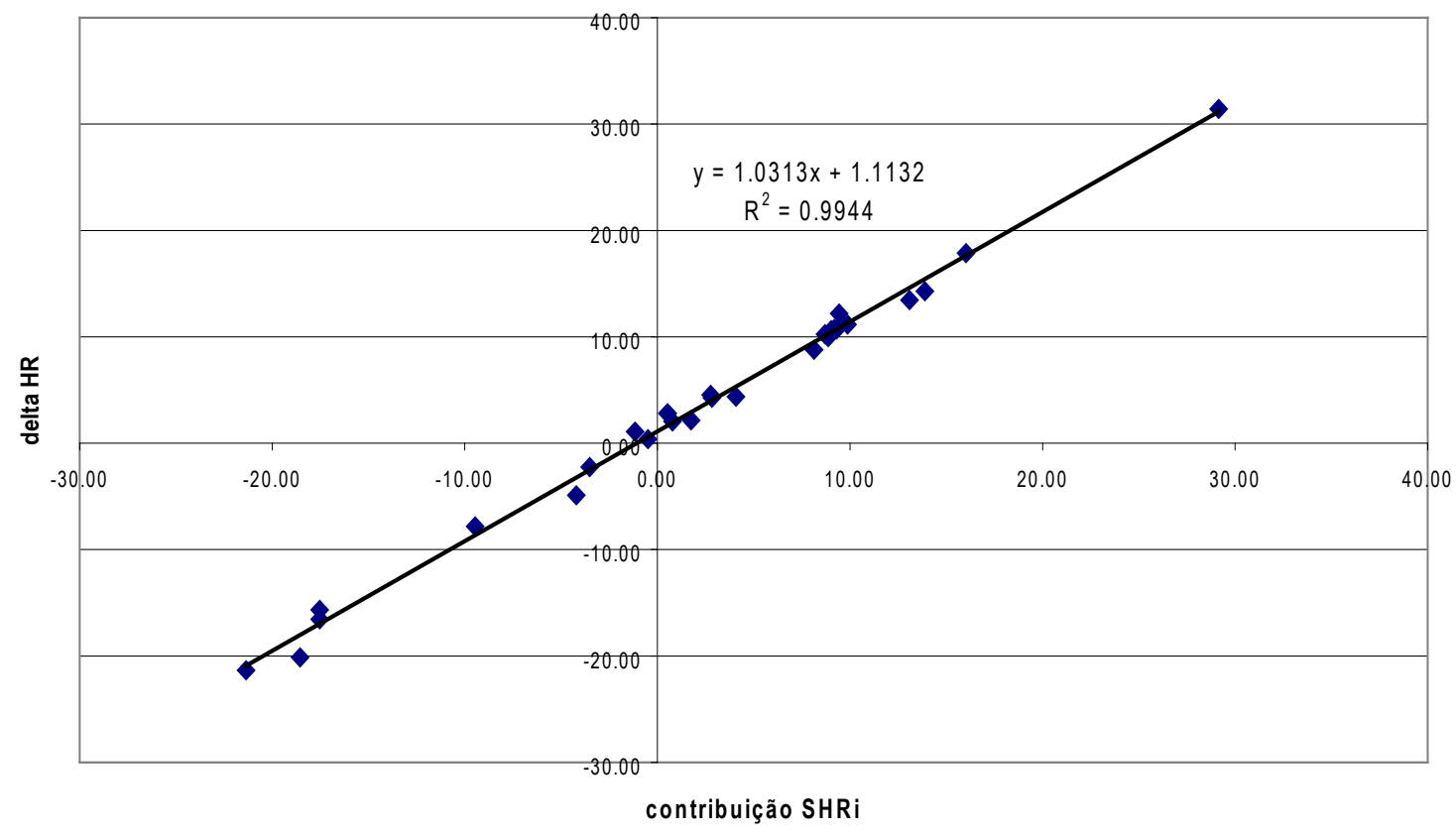

FONTE: Elaboração própria a partir dos dados do DATASUS.

Em resumo, podemos notar pelos Gráficos 4 e 5 que as alterações nas taxas específi- 
cas de mortalidade por homicídios no Brasil contribuem mais fortemente para a explicação das mudanças observadas nas taxas agregadas. Portanto, este trabalho mostrou que as mudanças nas condições econômicas e sociais, além de alterações nas variáveis que representam a repressão à violência, explicam de forma mais significativa a evolução (dependendo do caso, para cima ou para baixo) das taxas brutas de homicídios no Brasil no período entre 1996 a 2007 . Ao contrário da interpretação dos resultados de De Mello e Schneider (2007), nossos resultados sugerem que a "demografia" (estrutura etária) teve impacto bastante limitado na explicação da evolução da mortalidade por homicídios no Brasil, tal como em Levitt (1999).

\section{CONCLUSÃO}

Fatores demográficos são importantes para a compreensão de fenômenos socioeconômicos e para a formulação de políticas públicas. Este artigo tangenciou essas questões analisando o caso da criminalidade, mais especificamente, a taxa de homicídios no Brasil, no período de 1996 a 2007.

Em contraste com outro estudo feito para o Brasil - De Mello e Schneider (2007) - o artigo apresenta duas contribuições originais: (a) analisamos o fenômeno em nível nacional, e não apenas em São Paulo e; (b) utilizamos a decomposição da taxa de homicídios para verificar os impactos das mudanças na estrutura etária e na função mortalidade, permitindo uma visão mais precisa do que os autores citados chamam de efeito da "demografia".

Os resultados encontrados por aqueles autores, sobre a importância da contribuição total do grupo etário para explicar a queda da taxa de homicídios para São Paulo, são similares aos nossos. Entretanto, a decomposição da taxa mostra que, ao contrário do que afirmam os autores, o impacto da mudança na estrutura etária não é o fator principal em sua queda observada nos últimos anos: deve-se atentar, nesse caso, para as mudanças na função de mortalidade.

Em outras palavras, a demografia é, de fato, muito importante para se explicar o comportamento da taxa de homicídios no Brasil e o exercício feito aqui mostra como, se bem entendida, ela pode nos ajudar em nossa compreensão de problemas tão importantes como a criminalidade e uma de suas consequências mais sérias: os homicídios. 


\section{REFERÊNCIAS}

BECKER, G. S. Crime and punishment: an economic approach. Journal of Political Economy, Chicago, v. 76, n. 2, 1968.

DOS SANTOS, M. J.; KASSOUF, A. L. Estudos econômicos das causas da criminalidade no Brasil: evidências e controvérsias. Revista Economia, Brasília, v. 9, n. 2, 2008.

FOX, J. A. Trends in juvenile violence: a report to the U.S. attorney general on current and future rates of juvenile offending. Washington: Bureau of Justice Statistics, 1996. Relatório.

FOX, J.A. Trends in juvenile homicide, 1976-1996. Washington: Bureau of Justice Statistics, 1997. Relatório.

KITAGAWA, E. M. Components of a difference between two rates. Journal of the American Statistical Association, v. 50, n. 272, 1955.

LEVITT, S. D. The limited role of changing age structure in explaining aggregate crime rates. Criminology, v. 37, n. 3, 1999.

MELLO, J. M. P. de.; SCHNEIDER, A. Mudança demográfica e a dinâmica dos homicídios no estado de São Paulo. São Paulo em Perspectiva, São Paulo, v. 21, n. 1, 2007.

PRESTON, S. H.; HEUVELINE, P.; GUILLOT, M. Demography: measuring and modelling population process. Blackwell Publishing, 2001.

PHILIP, Y. Catching a coming crime wave - Profile: James Alan Fox. Scientific American, v. 274, n. 6, 1996.

WILSON, J. Q. What to do about crime? Commentary, v. 98, n. 3, 1994. 
